\title{
HERBAL REMEDY FOR OSTEOSARCOMA - CHALLENGING EVOLUTION
}

\author{
VIGNESH PICHAIYAN, SASIKALA MARIYAPPAN, RENUKA SARAVANAN, SIVAKUMAR RAMALINGAM*
}

Department of Chemistry and Biosciences, SASTRA Deemed to be University, Srinivasa Ramanujan Centre, Thanjavur - 612 001, Tamil Nadu, India. Email: rsiva@src.sastra.edu

Received: 07 March 2018, Revised and Accepted: 17 April 2018

ABSTRACT

Bone cancer is the extremely rare type, which accounts for about $0.2 \%$ of all cancer. They metastasize the cancer cells to the bone parts which have an especially crucial mechanism of action while treating. It is treated with the advanced drugs, have effluent side effects when compared to the medicinal plants and phytoconstituents. The review effectively deals that the mechanism of action of different medicinal plants include Phyllanthus urinaria, Aralia elata, Punica granatum, Anemone altaica, and Potentilla chinensis and the phytoconstituents include corosolic acid, shikonin, thymoquinone, aloperine, and withaferin A. This review provides the detailed information about the importance of medicinal herbs in the treatment of bone cancer, besides the mechanism of action of several phytoconstituents in different cell lines used. In future, it is more useful for evaluating the treatment of bone cancer with phytoconstituents.

Keywords: Osteosarcoma, Phytoconstituents, Medicinal plants.

(c) 2018 The Authors. Published by Innovare Academic Sciences Pvt Ltd. This is an open access article under the CC BY license (http://creativecommons. org/licenses/by/4. 0/) DOI: http://dx.doi.org/10.22159/ajpcr.2018.v11i7.25706

\section{INTRODUCTION}

Osteosarcoma (OS) is a pleomorphic malignant tumor of bone in which the proliferating spindle cells produce osteoid or immature bone [1]. It is a deadly form of musculoskeletal cancer that most commonly causes patients to die due to pulmonary metastatic disease [2]. It arises in bone during the periods of rapid growth, and it primarily affects adolescents and young adults. While incidence is low, it predominately affects the adolescents and young adults, and if untreated it becomes fatal. OS arises most commonly in the metaphyseal region of long bones, within the medullary cavity and penetrates the cortex of the bone to involve the surrounding soft tissues [3]. The most common sites are: Femur $(42 \%$ and $75 \%$ of which are in the distal femur), tibia $(19 \%$ and $80 \%$ of which are in the proximal tibia), humerus $(10 \%$ and $90 \%$ of which are in the proximal humerus) skull, and jaw (8\%), and pelvis (8\%). A number of variants of OS exist, including conventional types (osteoblastic, chondroblastic, and fibroblastic), telangiectatic, multifocal, parosteal, and periosteal [2]. Roughly 20\% of patients present with clinically detectable metastatic disease. Interestingly, several human genetic disorders and familial cancer syndromes, such as Li-Fraumeni syndrome are linked to an increased risk of OS [4].

Treatment generally includes pre-operative chemotherapy, surgical resection, and post-operative chemotherapy [5]. The importance of adjuvant chemotherapy for treatment of OS is well established [1]. Despite modern treatment protocols that combine chemotherapy, surgical operation, and sometimes radiotherapy, the 5-year survival rate for sufferers recognized with OS remains at $60-70 \%$ [3]. The mainstay of therapy is surgical removal of the malignant lesion. Most often, limb-sparing (limb keeping) technique can be used to treat sufferers with this disease and thus, preserves its function. Chemotherapy is also required to deal with micrometastatic disease, which is present but frequently no longer detectable in most sufferers $(\sim 80 \%)$ on the time of analysis [2]. Agents who have shown activity against OS include doxorubicin, cisplatin, and high-dose methotrexate with leucovorin rescue (HDMTX). All sufferers received identical cumulative doses of cisplatin, doxorubicin, and HDMTX, and underwent definitive surgical resection of the primary tumor [1]. Hence, there is a real need to optimize present treatment techniques and to increase novel methods for treating OS [3]. Present modes of treatment are based on synthetic drugs/chemotherapy has limited potential, because they may be toxic and expensive and also modify the functioning of the cell signaling pathways. The available anticancer drug is often unsatisfactory due to the problem, which causes cytotoxicity to the normal cells along with cancer cells. Plants are considered as the valuable sources of bioactive compounds with antioxidant activity, which produce certain substances that have effects on living animal cells [6]. Natural drugs which are safe, low cost and powerful are needed to control the cancer development and progression. Natural products have been used for thousands of years in the management of several diseases including various types of cancer. It is also known that herbal constituents may also play a key role in cancer treatment through antitumor activity or by suppressing bioactivation of carcinogen. There are numerous medicinal plants/ products which are beneficial for health and also have antitumor, antimicrobial, antibacterial, and antioxidant nature [7]

\section{PLANTS IN THE TREATMENT OF OS}

Chemotherapy is routinely used for cancer treatment. However, occasionally a number of undesired side effects and various kinds of toxicities may occur because of chemotherapeutic treatments [8]. Medicinal herbs and their derivative phytocompounds are being increasingly recognized as useful complementary treatments for different types of cancers [9]. Plant life has a long history of use in the treatment of cancer and played an important role as a source of effective anticancer agents [10]. 92 anticancer drugs commercially available before 1983 in the United States, approved worldwide between 1983 and 1994, approximately 62\% can be related to natural origins [11]. India is the largest manufacturer of medicinal plants and is rightly called the "Botanical garden of the World." Considerable works had been finished on these plants, and some plant products had been marketed as anticancer drugs, based on the traditional uses and scientific reports. The World Health Organization, about three-quarters of the world's population, currently uses herbs and other forms of traditional medicines to treat diseases [12].

The rhizomes of Anemone altaica. Fisch. ex C. A. Mey aqueous extract (AAE), suppressed the viability of human OS (HOS) and U2OS cells in a concentration-structured manner. Flow cytometry analysis revealed that AAE significantly improved the amount of cell shrinkage (Sub-G1 fragments) in HOS and U2OS cells. Moreover, AAE increased cytosolic cytochrome c and Bax but decreased B cell lymphoma-2 (Bcl-2). The 
amount of cleaved caspase- 3 and poly- (adenosine diphosphate [ADP]ribose) polymerase-1 (PARP-1) was significantly increased. AAE suppressed the growth of HOS and U2OS through the intrinsic apoptotic pathway. Thus, AAE has the great capacity as a useful therapeutic drug for HOS [13].

Aralia elata is a well-known traditional Chinese herbal medicine. MTT assays confirmed that a polysaccharide (acute eosinophilic pneumonia [AEP]-1) from the leaves of $A$. elata markedly inhibited the growth of U-2 OS cells in a dose- and time-structured fashion, suggesting a cytotoxic effect and also dose-dependently induced DNA fragmentation and induced apoptotic death in U2OS cells through the increased ratio of Bax/Bcl-2, depolarization of mitochondrial membrane ability and the release of cytochrome $\mathrm{c}$ from mitochondria into the cytoplasm. Moreover, AEP-1 treatment induces the activation of caspase- 9 and caspase-3, as well as the cleavage of poly (ADP-ribose) polymerase (PAPR) in U-2 OS cells. Thus, those findings suggested that AEP-1 probably have an important potential as the most cancer preventive and therapeutic agent toward HOS [14].

Oldenlandia diffusa (OD) is a well-known traditional Chinese medicine, which is used to prevent and treat many disorders, particularly cancers. OD inhibited proliferation and triggered apoptosis inside the HOS MG63 cell line in a time-dependent and dose-based manner. In addition, OD displayed inhibitory activity on MG-63 cell proliferation and invasion, and the study also showed that OD activity probably mediated with the aid of caspase activation. Consequently, the study suggested that OD might represent a novel, efficient candidate agent for OS treatment [15].

Phyllanthus urinaria is widely used as anti-inflammatory, antiviral, antibacterial, and anti-hepatotoxic medicines. The HOS Saos-2 cell line, and looked at the effect of $P$. urinaria extract (PUE) on several relevant proteases and signaling pathways. This study demonstrates that PUE, at various concentrations (from 0 to $100 \mathrm{mg} / \mathrm{ml}$ ), awareness-dependently inhibited the migration/invasion capacities of Saos-2 without cytotoxic effects. Western blot analysis also confirmed that PUE inhibits phosphorylation of extracellular signal-regulated kinase (ERK) $1 / 2$ and AKT the inhibitory effects of PUE on u-PA expression in Saos- 2 cells. The chromatin immunoprecipitation assay changed into reactive to the transcription protein SP-1, which became inhibited by means of PUE. It suppresses HOS Saos-2 cell invasion and migration by transcriptionally inhibiting u-PA through ERK and AKT signaling pathways. Therefore, PUE produces anti-metastatic activity in Saos-2 cells [16].

Pomegranate peels (Punica granatum L.), one of the most valuable by-products of the food industry, have attracted lot attention due to its wide range of bioactivities. Polysaccharide from pomegranate peels (PPP) was found to induce the arrest of G2/M segment, induce apoptosis, and inhibit the growth of U-2 OS (OS) cells in a dose-based way. Western blotting analysis showed that PPP precipitated the mitochondrial apoptotic pathway, as indicated through a growth in $\mathrm{Bax} / \mathrm{Bcl}-2$ ratios, a loss of mitochondrial membrane potential, release of cytochrome c, activation of caspase- 9 and caspase-3, and cleavage of PARP in U-2 OS cells. Hence, the study suggested that PPP might be a strong chemotherapeutic agent against OS [17].

The ethanol extracts of Potentilla chinensis triggered early and late apoptosis within the most cancer cells. Flow cytometric analysis revealed that the extract triggered G0/G1-cell cycle arrest, which also showed significant dose-dependent and caused a significant and concentration-dependent reduction in cell migration. Hence, the extract exhibited a selective cytotoxic effect against MG-63 OS cells, in order that it serves as a novel therapeutic agent toward OS [18].

Rheum palmatum L. (Dahuang) is a wild rebus, erect, glabrous, and perennial aromatic herb. The crude extract of R. palmatum L. (CERP) substantially brought about cytotoxic effects in U-2 OS HOS cancer cells through $S$ phase arrest and induce nuclear DNA damage and condensation were seemed to activate cysteine proteases of the caspase family; such as caspase- $3,-8$, and -9 , and the induction of apoptosis to be correlated with the caspase-dependent pathway. CERP increased the levels the ranges of Bax, Bak, Bad, cyclin B, Fas, PARP, GRP78, GADD153, apoptosis-inducing factor (AIF), Endo G, Calpain-2, p21, and p27, but decreased the levels of $\mathrm{Bcl}-2, \mathrm{Bcl}-\mathrm{X}, \mathrm{X}$-linked inhibitor of apoptosis (XIAP), AKT, cell division cycle 25 homolog A, cyclin-dependent kinase 2 (CDK2), cyclin A, and cyclin E of U-2 OS cells. It was also observed that CERP promoted the expression of AIF, Endo G, GADD153, and cytochrome $c$. Those results indicate that CERP has anticancer activity on OS cells $[19,20]$.

Sarcandra glabra (Thunb.) Nakai is a plant belonging to the own family of Chloranthaceae which exhibits anti-cancer activities. An ethyl acetate (EA) extract of $S$. glabra suppresses the proliferation of human leukemic -60 cells by means of upregulating the pro-apoptotic Bax/Bcl-2 ratio associated with cell cycle arrest. Hence, these results suggest that SGP-2 has anticancer potential in the treatment of HOS [21].

Selaginella tamariscina is a traditional medicinal plant for treatment of some advanced cancers within the orient. It possesses antiinflammatory, antibacterial, antihypertensive, and anti-hyperglycemic activities. Zymographic and western blot analyses revealed that S. tamariscina inhibited the matrix metalloproteinase (MMP)-2 and MMP-9 enzyme activity, as well as protein expression. Western blot analysis also showed that it possesses an antimetastatic activity in OS cells by way of downregulating MMP-2 and MMP-9 secretions and growing tissue inhibitor of metallopeptidases (TIMP)-1 and TIMP-2 expressions through p38 and AKT-structured pathways and may be a powerful candidate to expand a preventive agent for OS metastasis [22].

Viscum album L. extracts are widespread in complementary most cancers medicine in Europe and used in several cancer types. V. album L. includes a large number of chemically special substances: Lectins, triterpenic acids, viscotoxins, phenolic acids, flavonoids, oligo- and polysaccharides. A whole mistletoe extract Viscum TT re-created by combining an aqueous extract (viscum) and a triterpene (TT) extract was tested for its anticancer ability in OS. The whole mistletoe extract Viscum TT led to strong inhibition of proliferation and synergistic apoptosis induction in OS cells. In the investigations of the mechanism of action, inhibitors of apoptosis which includes XIAP, BIRC5, and CLSPN showed a clear downregulation after Viscum TT treatment. In addition, coremedy with doxorubicin, etoposide, and ifosfamide further enhanced apoptosis induction, also synergistically. Hence, Viscum TT may represent a promising adjuvant therapy in pediatric OS [23].

\section{PHYTOCONSTITUENTS IN THE TREATMENT OF OS}

Natural products are commonly safe, effective, and less expensive substitutes of anticancer chemotherapeutics [7]. Natural therapies, such as the use of plant-derived products in most cancer treatment, can also reduce adverse side effects [8]. Several reports describe that the anticancer activity of medicinal plants is due to the presence of antioxidants in them. In fact, the medicinal plants are easily available, cheaper, and possess no toxicity as compared to the modern (allopathic) drugs. Secondary metabolites such as polyphenols, terpenes, and alkaloids have been suggested to possess antimutagenic and anticancer properties in many research [12]. Plant-derived compounds had been an important source of several clinically useful anticancer agents. Natural phenolic compounds play an important role in cancer prevention and treatment. Various bioactivities of phenolic compounds are responsible for their chemopreventive properties (e.g., antioxidant, anticarcinogenic, or antimutagenic, and anti-inflammatory effects) [24].

Aloperine (ALO) is a novel alkaloid drug extracted from S. alopecuroides and exerts anti-inflammatory, anti-allergenic, antitumor, and antiviral effects. DAPI assays and flow cytometry showed that ALO induced apoptosis of OS cells. Moreover, the outcomes of western blotting and quantitative real-time polymerase chain reaction indicated that ALO upregulated protein and mRNA of Bax and cleaved caspase-3, while downregulated Bcl-2. Besides, ALO inhibited the invasion of MG-63 and 
U2OS cells through suppression of PI3K/AKT signaling pathway on OS cells [25].

Astragaloside IV (3-0-beta-D-xylopyranosyl-6-0-beta-D-glucopyranosylcycloastragenol), a potent ingredient extracted from Astragalus membranaceus Bunge. It suppressed cell proliferation and enhanced chemosensitivity in OS cell lines and xenograft. Caspase-dependent Fas/ FasL signaling turned into worried in cisplatin brought on apoptosis, which becomes greater by Astragaloside IV. It indicated that Astragaloside IV might be a promising therapeutic agent for OS treatment [26].

Berberine (BBR), an isoquinoline alkaloid component aspect in several Chinese herbs including Huang lian, that have antimicrobial, antiinflammatory, anti-diabetic, and anti-angiogenesis, and cholesterol reducing effects. The proliferation effect of U20S changed into examined by MTT assay and the percentage of apoptotic cells was determined by flow cytometric analysis. This treatment caused dosestructured inhibiting proliferation and inducing apoptosis of U20S cell through inhibiting the PI3K/AKT signaling pathway activation results in upregulating the expression of Bax, and PARP and downregulating the expression of Bcl-2 and caspase 3 . Thus, the study supports that BBR might be a good alternative therapy for treatment of OS within the clinical practice [27].

Columbamine (COL), an active component of the herb Coptis chinensis, inhibited the proliferation and neovascularization of metastatic OS U2OS cells. It's effectively suppressed U2OS cell proliferation in vitro with an inhibitory concentration 50 of $21.31 \pm 0.38 \mu \mathrm{M}$, with low cytotoxicity. Mechanistic studies revealed that COL induces cell cycle arrest at the G2/M transition that is related to attenuating CDK6 gene expression and diminishing STAT3 phosphorylation and did not promote U2OS cell apoptosis at any of the dosages tested. In addition, COL inhibited U20S cell-mediated neovascularization, which was observed by the downregulation of MMP 2 expression and reduction of cell migration, adhesion, and invasion. Hence, the study showed that COL exerts antiproliferative and anti-vasculogenic effects on metastatic HOS U2OS cells with low toxicity and used as a potential anti-OS and anticancer drug [28].

Coptisine, an active component of the herb Coptidis rhizoma, markedly inhibited aggressive OS cell proliferation. Coptisine induced cell cycle arrest at the G0/G1 phase through downregulation of CDK4 and cyclin D1 expression and effectively suppressed tumor growth. Coptisine appreciably impeded OS cell migration, invasion, and capillary-such as network formation by decreasing the expression of VE-cadherin, integrin-3, and diminishing STAT3 phosphorylation. Thus, these data suggest that coptisine exerts a strong anti- OS effect with very low toxicity and is an ability anti-OS drug candidate [29].

Corosolic acid (CRA), a pentacyclic TT isolated from medicinal herbs, consisting of Schisandra chinensis, Lagerstroemia speciosa, Orthosi phonstamineus, and Eriobotrya japonica. It suppresses the growth of various types of tumors, the inhibitory effect of CRA in OS MG-63 cells was investigated, and the results revealed that it significantly inhibited the viability of MG-63 cells in a dose- and time-established way. It was observed that the apoptosis of MG-63 cells induced by CRA was closely related to activation of caspase- 3 and caspase- 9 , loss of mitochondrial membrane ability. Therefore, those results indicated that CRA should induce apoptosis of OS cells through activating the mitochondrial pathway [30].

Cucurbitacin E ( $\mathrm{CuE}$ ), an amazing member of triterpenoid family isolated from plants, has been confirmed as an antitumor agent using inhibiting proliferation, migration, and metastasis in diverse cancer. The present study aimed to test whether CuE could inhibit increase and invasion of OS cells and monitoring its underlying molecular mechanism. After various concentrations of $\mathrm{CuE}$ treatment, the anti-proliferative effect of $\mathrm{CuE}$ became assessed using the cell counting Kit-8 assay. Cell cycle distribution was analyzed by propidium iodide staining. CuE inhibited cell growth and invasion, induced a cell cycle arrest and triggered apoptosis and modulated the expression of cell growth, cell cycle, and cell apoptosis regulators. CuE inhibited the PI3K/AKT/mTOR pathway and epithelial-mesenchymal transition, which suppressed the invasion and metastasis of OS. Our study demonstrated that $\mathrm{CuE}$ could inhibit OS tumor growth and invasion through inhibiting the PI3K/AKT/mTOR signaling pathway. Our findings suggest that $\mathrm{CuE}$ can be considered to be a promising anticancer agent for OS [31].

Curcumin, an active factor derived from the rhizome of the plant, Curcuma longa, has antioxidant, anti-inflammatory, and anticancer properties. Curcumin confirmed the growth of inhibitory effects on U2OS cells in a dose- and time-structured way, inducing significant G1 arrest and apoptosis in U2OS cells. This curcumin-induced apoptosis in U2OS cells was accompanied by upregulation of Bax, Bak, and p-Bad and downregulation of Bcl-2, however, no effect on the levels of Bcl-XL or Bad proteins were noted. Moreover, curcumin treatment resulted in a significant reduction of mitochondrial membrane ability and increase in the concentrations of mitochondrial cytochrome C and caspase-3 [32].

5,7dihydroxy3',4',6trimethoxy flavone, normally known as eupatilin, is extracted from Artemisia asiatica Nakai and consists of pharmacologically active ingredients and has been established to exert anticancer, antioxidative, and anti-inflammatory effects. The experimental results revealed that eupatilin inhibited U2-OS cell growth in a concentration-based manner and induced G2/M phase cell cycle arrest and apoptosis. Western blot analysis indicated that it was able to cause the mitochondrial apoptotic pathway, by the enhanced Bax/Bcl-2 ratio, decrease in mitochondrial membrane capability, the release of cytochrome $c$, caspase- 3 and -9 activation and PAPR cleavage detected in the U2-OS cells. Hence, eupatilin may, therefore, represent a novel anticancer drug for use in the treatment of OS [33].

The polyphenolic fraction of green tea (green tea polyphenols [GTP]) has been shown to have antitumor effects on various malignant cell lines to inhibit growth and induce apoptosis in HOS SAOS-2 cells. Treatment of SAOS- 2 cells with GTP (20-60 $\mu \mathrm{g} / \mathrm{ml})$ resulted in reduced cell proliferation and induction of apoptosis, which correlated with decreased nuclear DNA binding of nuclear factor kappa (NF- $\kappa \mathrm{B}) /$ p65 and lowering of NF- $\mathrm{kB} / \mathrm{p} 65$ and p50 levels in the cytoplasm and nucleus. This treatment of cells has reduced IкB- $\alpha$ phosphorylation but had no effect on its protein expression. Furthermore, this treatment resulted in the inhibition of I $\mathrm{KK}-\alpha$ and I $\mathrm{KK}-\beta$, the upstream kinases that phosphorylate I $\mathrm{KB}-\alpha$. The increase in apoptosis in SAOS- 2 cells was accompanied by a decrease in the protein expression of Bcl-2 and a concomitant increase in the levels of Bax. This treatment of SAOS2 cells also resulted in significant activation of caspases as evident by increased levels of cleaved caspase- 3 and caspase- 8 in these cells. Hence, these studies indicate that GTP is a candidate therapeutic for OS [34].

Plumbagin (5-hydroxy-2-methyl-1,4-naphtho quinone), a yellow pigment is natural occurring quinonoid constituent of Plumbago zeylanica L. (Plumbaginaceae). It exhibits various biological activities such as antiatherogenic, anticancer, anti-proliferative, cardiotonic, chemoprevention, hepatoprotective, and neuroprotective effects. It also exhibits pro-apoptotic and radio-sensitizing activities in distinct tumor cells. It has significantly induced growth inhibition against OS MG-63 cells, primarily by S-phase cell cycle arrest which is confirmed by the downregulation of cyclin A and CDK2 protein levels determined by western blot analysis. It was also found that plumbagin has brought out the DNA damage in MG-63 cells, subsequently initiating the arrest in S-phase, which is evident by the upregulation of phosphorylated p53 and histone. Thus, the study suggested that it to be a potential compound in treatment in opposition to the malignant HOS [35].

Polyphyllin I (PPI) is an ethanol extraction from Paris polyphylla Smith var. yunnanensis. PPI inhibited viability, proliferation, migration, and invasion of MG-63, Saos-2, and U-2 OS OS cells, and led to S-phase arrest 
and apoptosis. Exposure of OS cells to PPI triggered inactivation of the intrinsic nuclear component $\kappa \mathrm{B}(\mathrm{NF}-\mathrm{\kappa B})$ and activation of unfolded protein response/endoplasmic reticulum stress signaling cascade in OS cells, followed by downregulation of anti-apoptotic proteins, with up regulation of pro-apoptotic proteins. Thus, it can be used for OS treatment [36].

Resveratrol (3,5,4'-trihydroxy-trans-stilbene) is a natural polyphenol compound, widely found in plants such as grape skin, raspberries, mulberries, cranberries, blueberries, and peanuts which have been widely reported as an anticancer molecule. It possesses antioxidative, anti-inflammatory, anti-aging, antiviral, and anticancer properties. It also exhibits multiple tumor-suppressing activities in bone cancer by affecting a series of critical events and has been found very effective in inhibiting proliferation in HOS cells by means attenuating the $\beta$-catenin signaling and also inducing apoptosis [37].

Sodium selenite (Na2Se03, SSE) is an inorganic Se compound that is widely used in most cancers chemoprevention studies. SSE also inhibited cell viability through apoptosis, as evidenced by the formation of apoptotic bodies, generation of reactive oxygen species (ROS), and accumulation of cells at some point of the advanced phase of apoptosis. SSE-triggered apoptosis correlated with the activation of CASP 3, downregulation of Bcl-2, and upregulation of P53, and PTEN in U20S cells. Thus, the studies state that SSE is a promising anticancer compound that would be used in future therapies for OS [38].

Solamargine (SM), a steroidal glycoalkaloid isolated from the Chinese herb Solanum incanum. It appreciably reduced cell viability and induced apoptosis in OS U2OS cell. Thus, increases the mRNA and protein expressions of p53 and Bax. The expression of Bcl-2 (an antiapoptotic protein) was also reduced. Furthermore, SM triggered mitochondrial translocation of p53, loss of mitochondrial membrane capacity, cytochrome c release activation of caspase- 9 and -3 , and induced apoptosis. Therefore, the study suggested that SM is a powerful inducer of apoptosis through p53 activation and may use as a potential therapeutic agent for OS [39].

Shikonin (SK), an active constituent extracted from a Chinese medicinal herb, Lithospermum erythrorhizon, has been shown to exert antitumor effects. Western blot analysis revealed increased expression levels of Bax, caspase-3, caspase-8, and PARP in U2OS and MG63 cells with the treatment of SK and ADM. Flow cytometric analysis showed that the combined treatment of SK and ADM significantly precipitated apoptosis probably by inducing caspase- 3 and caspase- 8 dependent apoptosis in the OS cells and maybe ability enhancer in the treatment of drugresistant primary OS [40].

Tanshinone IIA (Tan IIA) is an active ingredient extracted from the widely used Danshen root (Salvia miltiorrhiza Bunge), a traditional Chinese medicine. It inhibits proliferation and induces apoptosis in the HOS cell line MG-63 in a time-dependent and dose-based way. In addition, it displays inhibitory activity on OS cell migration and invasion. Mechanistic studies show that Tan IIA activity is mediated by caspase activation and it was also shown to reduce anti-apoptotic Bcl-2, MMP-2, and MMP-9 levels, whereas it increased proapoptotic Bax levels. These data suggest that Tan IIA may be a novel, efficient candidate agent for OS treatment [41].

Thymoquinone (TQ), the predominant bioactive constituent derived from the medicinal spice Nigella sativa (also known as black cumin), it caused a higher percentage of growth inhibition and apoptosis within the HOS cell line SaOS-2 compared to that of control in a dosedependent manner. The EMSA assay and western blot evaluation found that the TQ significantly downregulated NF- $\mathrm{KB}$ DNA-binding activity, XIAP, surviving, and VEGF in SaOS-2 cells, and inhibits tumor angiogenesis and tumor growth through suppressing NF- $\mathrm{KB}$ and its regulated molecules on SaOS-2 cells [42].
Withaferin A (WA) is a well-known steroidal lactone of the medicinally important plant, Withania somnifera. They are reported to possess anticancer properties. Flow cytometric analysis revealed that WA induced cell cycle arrest at the G2/M phase, which was associated with the inhibition of cyclin B1, cyclin A, CDK2, and p-Cdc2 (Tyr15) expression, an increase in the levels of p-Chk1 (Ser345) and p-Chk2 (Thr68) and attenuation of the expression levels of G2/M checkpoint proteins. Hence, the results revealed that WA exhibited potent antiproliferative effects on the HOS cell lines, MG-63, and U2OS [43].

Wogonin (5,7-dihydroxy-8-methoxyflavone) is a flavone constituent of Scutellaria baicalensis with various beneficial biological activities, and it has been shown to have tumor healing ability in vitro and in vivo. Flow cytometric assay indicated that it precipitated sub- $G_{1}$ phase (apoptosis) and increased caspase- 3 activity in examined cells. It also brought about apoptosis in U-2 OS cells was also confirmed by DAPI staining. Furthermore, results from Western blotting indicated that it extended the levels of Bad, Bax, cytochrome $c$, cleaved caspase-9, cleaved caspase-3, AIF, Endo G, Fas/CD95, caspase-8, GADD153, GRP78, ATF$6 \alpha$, calpain 1 , calpain 2 , and caspase- 4 then leading to cell apoptosis. Thus, the study suggests that wogonin could be developed for the treatment of HOS in the future [44].

\section{CONCLUSION}

For the betterment, while treating cancer with the drugs, it is more effective when treated with the medicinal plants. Thus, the above review replenishes the usage of the medicinal herbs for especially the treatment of bone cancer, and it has the potential access to cancer treatment

\section{AUTHOR'S CONTRIBUTION}

Sivakumar Ramalingam has provided the design, intellectual content, innovations, and protocol for the review article. VigneshPichaiyan has majorly performed the review. Sasikala Mariyappan and Renuka Saravanan have a minor role in performing the review, and sincerely authored the article.

\section{CONFLICTS OF INTEREST}

The authors declare that there are no conflicts of interest regarding the publication of this article.

\section{REFERENCES}

1. Meyers PA, Schwartz CL, Krailo M, Kleinerman ES, Betcher D, Bernstein ML, et al. A randomized, prospective trial of the addition of ifosfamide and/or muramyltripeptide to cisplatin, doxorubicin, and high-dose methotrexate. J Clin Oncol 2005;23:1-9.

2. Campanacci M. Bone and soft tissue tumors: Clinical features, imaging, pathology and treatment. $2^{\text {nd }}$ ed., New York: Springer-Verlag;1999:5-90.

3. Broadhead ML, Clark JC, Myers DE, Dass CR, Choong PF. The molecular pathogenesis of Osteosarcoma. Hindawi Publ $2011 \cdot 2011: 1-12$.

4. Gorlick R, Khanna C. Osteosarcoma. J Bone Miner Res 2010;25:683-91.

5. Messerschmitt PJ, Garcia RM, Abdul-Karim FM, Greenfield EM, Getty PJ. Osteosarcoma. J Am Acad Orthop Surg 2009;17:515-27.

6. Aishwarya V, Abdulla S, Dheeba B, Renuka R. In vitro antioxidant and anticancer activity of Cardiospermum halicacabum L. against EAC cell line. Int J Pharmacy and Pharm Sci 2014;6:263-8.

7. Rahmani AH, Alzohairy MA, Khan MA, Aly SM. Therapeutic implications of black seed and its constituent thymoquinone in the prevention of cancer through inactivation and activation of molecular pathways. Evid Based Complement Altern Med 2014;2014:1-13.

8. Desai AG, Qazi GN, Ganju RK, Tamer ME, Singh J, Saxena AK, et al. Medicinal plants and cancer chemoprevention. Curr Drug Metab 2008;9:581-91.

9. Yin SY, Wei WC, Jian FY, Yang NS. Therapeutic applications of herbal medicines for cancer patients. Hindawi Publishing Corporation. Evid Based Complement Altern Med 2013;2013:1-15.

10. Cragg GM, Newman DJ. Plants as a source of anti-cancer agents. J Ethnopharmacol 2005;100:72-9.

11. Prasanth NV, Dilip C, Dev S, Augustine L, Saraswathi R. Evaluation 
of in vitro cytotoxic and antioxidant activities of Ipomoea batatas. Int $\mathrm{J}$ Pharm Pharm Sci 2010;2:91-2.

12. Umadevi M, Kumar KP, Bhowmik D, Duraivel S. Traditionally used anticancer herbs in India. J Med Plants Stud 2013;1:56-74.

13. Chang IC, Chiang TI, Lo C, Lai YH. Anemone altaica induces apoptosis in human osteosarcoma cells. Am J Chin Med 2015;43:1-12.

14. Liua G, Sheng Y, Zhang M, Sun D. A polysaccharide from the leaves of Aralia elata induces apoptosis in U-2 OS cells via mitochondrialdependent pathway. Int J Biol Macromol 2016;93:418-25.

15. $\mathrm{Pu} \mathrm{F}$, Chen F, Lin S, Chen S. The synergistic anticancer effect of cisplatin combined with Oldenlandia diffusa in osteosarcoma MG-63 cell line in vitro. Dove Press J 2016;9:255-63.

16. Lu KH, Yang HW, Su CW, Lue KH. Phyllanthus urinaria suppresses human osteosarcoma cell invasion and migration by transcriptionally inhibiting u-PA via ERK and AKT signaling path-ways. Food Chem Toxicol 2013;52:193-9.

17. Li J, Zhang F, Wang S. A polysaccharide from pomegranate peels induces the apoptosis of human osteosarcoma cells via the mitochondrial apoptotic pathway. Tumour Biol 2014;35:7475-82.

18. Wan $\mathrm{G}$, Tao JG, Wang GD, Liu SP, Zhao $\mathrm{HX}$, Liang $\mathrm{QD}$, et al. In vitro antitumor activity of the ethyl acetate extract of Potentilla chinensis in osteosarcoma cancer cells. Mol Med Rep 2016;14:3634-40.

19. Miraj S. Therapeutic effects of Rheum palmatum L. (Dahuang): A systematic review. Der Pharm Chem 2016;8:50-4.

20. Lin CC, Lee MH, Lin JH, Lin ML. Crude Extract of Rheum palmatum L. Induces cell cycle arrest $\mathrm{S}$ phase and apoptosis through mitochondrialdependent pathways in U-2 OS human osteosarcoma cells. Wiley Periodicals Inc. Environ Toxicol 2014;31:957-69.

21. Zhang Z, Liu W, Zheng Y, Jin L, Yao W, Gao X. SGP-2, an acidic polysaccharide from Sarcandra glabra, inhibits proliferation and migration of human osteosarcoma cells. R Soc Chem 2014;5:165-75.

22. Yang JS, Lin CW, Hsieh YS, Cheng HL, Lue KH, Yang SF, et al. Selaginella tamariscina (Beauv.) possesses antimetastatic effects on human osteosarcoma cells by decreasing MMP-2 and MMP9 secretions via $\mathrm{p} 38$ and AKT signaling pathways. Food Chem Toxicol 2013;59:801-7.

23. Kleinsimon S, Kauczor G, Jaeger S, Eggert A, Seifert G, Delebinski C. Viscum TT induces apoptosis and alters IAP expression in osteosarcoma in vitro and has synergistic action when combined with different chemotherapeutic drugs. BMC Complement Altern Med 2017;17:1-26.

24. Huang WY, Cai YZ, Zhang Y, Natural phenolic compounds from medicinal herbs and dietary plants: Potential use for cancer prevention. Routledge. Nutr Cancer 2009;62:1-20.

25. Chen S, Jin Z, Dai L, Wu H. Aloperine induces apoptosis and inhibits invasion in MG-63 and U2OS human osteosarcoma cells. Biomed Pharmacother 2018;97:45-52.

26. Hu T, Fei Z, Wei N. Chemosensitive effects of astragaloside IV in osteosarcoma cells via induction of apoptosis and regulation of caspasedependent fas/FasL signaling. Pharmacol Rep 2017;69:1159-64.

27. Chen Z. Berberine induced apoptosis of human osteosarcoma cells by inhibiting phosphoinositide 3 kinase/protein kinase B (PI3K/AKT) signal pathway activation. Iran J Public Health 2016;45:578-85.

28. Bao M, Cao Z, Yu D, Fu S. Columbamine suppresses the proliferation and neovascularization of metastatic osteosarcoma U2OS cells with low cytotoxicity. Toxicol Lett 2015;215:174-80.

29. Yu D, Fu S, Cao Z, Bao M. Unraveling the novel anti-osteosarcoma functionofcoptisine and its mechanisms. Toxicol Lett 2014;226:328-36.

30. Jia Y, Yuan H, Shan S, Xu G. Corosolic acid inhibits the proliferation of osteosarcoma cells by inducing apoptosis. Oncol Lett 2016;12:4187-94.

31. Wang Y, Xu S, Wu Y, Zhang J. Cucurbitacin E inhibits osteosarcoma cells proliferation and invasion through attenuation of $\mathrm{PI} 3 \mathrm{~K} / \mathrm{AKT} /$ mTORsignaling pathway. Biosci Rep 2016;36:1-11.

32. Jin S, Xu HG, Shen JN, Chen XW. Apoptotic effects of curcumin on human osteosarcoma U2OS cells. Orthop Surg 2009;1:144-52.

33. Li YY, Wu H, Dong YG, Lin B, Xu G, Ma YB, et al. Application of eupatilin in the treatment of osteosarcoma. Oncol Lett 2015;10:2505-10.

34. Hafeez BB, Ahmed S, Wang N, Gupta S, Zhang A, Haqqi TM. Green tea polyphenols-induced apoptosis in human osteosarcoma SAOS2 cells involves a caspase-dependent mechanism with downregulation of nuclear factor- $\kappa$ B. Toxicol Appl Pharmacol 2006;216:11-9.

35. Yan CH, Li F, Ma YC. Plumbagin shows anticancer activity in human osteosarcoma (MG-63) cells via the inhibition of S-Phase checkpoints and down-regulation of c-myc. Int J Clin Exp Med 2015;8:14432-9.

36. Chang J, Wang H, Wang X, Zhao Y. Molecular mechanisms of Polyphyllin I-induced apoptosis and reversal of mesenchymal transition in human osteosarcoma cells. J Ethno Pharmacol 2015;170:117-27.

37. Chen G, Xia H, Zhang ZG, Yu HL. Resveratrol in management of bone and spinal cancers. Nat Prod Res 2017;2017:1-11.

38. Chen XJ, Duan FD, Zhang HH, Xiong Y, Wang J. Sodium seleniteinduced apoptosis mediated by ROS attack in human osteosarcoma U2OS Cells. Biol Trace Elem Res 2012;145:1-9.

39. Li X, Zhao Y, Wu WKK, Liu S, Cui M, Lou H. Solamargine induces apoptosis associated with p53 transcription-dependent and transcription-independent pathways in human osteosarcoma U2OS cells. Life Sci 2011;88:314-21.

40. Yang Q, Li S, Fu Z, Lin B, Zhou Z, Wang Z, et al. Shikonin promotes adriamycin-induced apoptosis by upregulating caspase-3 and caspase- 8 in osteosarcoma. Mol Med Rep 2017;16:1347-52.

41. Zhang Y, Wei RX, Zhu XB, Cai L. Tanshinone IIA induces apoptosis and inhibits the proliferation, migration, and invasion of the osteosarcoma MG-63 cell line in vitro. Anti Cancer Drugs 2012;23:212-9.

42. Peng L, Liu A, Shen Y, Zi XU. Antitumor and anti-angiogenesis effects of thymoquinone on osteosarcoma through the NF- $\kappa$ B pathway. Oncol Reports 2013;29:571-8.

43. Zhuo LV, Wang GS. Antiproliferation potential of with aferin A on human osteosarcoma cells via the inhibition of $\mathrm{G} 2 / \mathrm{M}$ checkpoint proteins. Exp Ther Med 2015;10:323-9.

44. Lin CC, Kuo CL, Lee MH, Lai KC. Wogonin triggers apoptosis in human osteosarcoma U-2 OS cells through the endoplasmic reticulum stress, mitochondrial dysfunction and caspase-3-dependent signaling pathways. Int J Oncol 2011;39:217-24. 